Meta

Journal des tradlucteurs

Translators' Journal

\title{
Glossaire de la théorie interprétative de la traduction et de l'interprétation
}

\section{Monique C. Cormier}

Volume 30, numéro 4, décembre 1985

URI : https://id.erudit.org/iderudit/002383ar

DOI : https://doi.org/10.7202/002383ar

Aller au sommaire du numéro

Éditeur(s)

Les Presses de l'Université de Montréal

ISSN

0026-0452 (imprimé)

1492-1421 (numérique)

Découvrir la revue

Citer ce document

Cormier, M. C. (1985). Glossaire de la théorie interprétative de la traduction et de l'interprétation. Meta, 30(4), 353-359. https://doi.org/10.7202/002383ar d'utilisation que vous pouvez consulter en ligne.

https://apropos.erudit.org/fr/usagers/politique-dutilisation/ 


\section{GLOSSAIRE DE LA THÉORIE INTERPRÉTATIVE DE LA TRADUCTION ET DE L'INTERPRÉTATION*}

Le présent glossaire regroupe les principaux termes utilisés dans la théorie interprétative de la traduction et de l'interprétation élaborée par Danica Seleskovitch et l'équipe de recherches de l'ÉSIT à l'Université Paris III/Sorbonne nouvelle. Il vise à préciser certaines notions fondamentales à l'intention des chercheurs qui s'intéressent à cette théorie.

Les termes qui figurent dans ce glossaire sont accompagnés de leur définition. La note précédée d'un astérisque qui suit parfois la définition d'un terme apporte des renseignements complémentaires. Par ailleurs, lorsqu'un terme comporte un ou plusieurs synonymes, ceux-ci sont indiqués. Enfin, dans certains cas, la mention V. (voir) renvoie à un autre terme où l'on trouvera la définition recherchée tandis que la mention V.a. (voir aussi) renvoie à un ou plusieurs termes qui entretiennent une relation avec le terme traité.

\section{ANTICIPATION COGNITIVE \\ V. ANTICIPATION DU SENS}

\section{ANTICIPATION DE LANGUE}

V. ANTICIPATION LINGUISTIQUE

\section{ANTICIPATION DU SENS}

Mouvement de la pensée qui permet d'anticiper le sens d'un discours ou d'un texte grâce aux éléments d'information fournis par le début de ce discours ou de ce texte.

* C'est la présence d'un contexte cognitif qui permet à l'anticipation du sens de se réaliser.

Synonymes : ANTICIPATION COGNITIVE

ANTICIPATION SENSIQUE

V.a. ANTICIPATION INTELLECTUELLE

\section{ANTICIPATION INTELLECTUELLE}

Mouvement de la pensée qui permet d'anticiper la direction que prendra un discours ou un texte après que l'interprète ou le traducteur aura pris connaissance du début de ce discours ou de ce texte.

V.a. ANTICIPATION DU SENS

\section{ANTICIPATION LINGUISTIQUE}

Mouvement de la pensée qui permet d'anticiper un mot en l'associant au précédent.

* L'anticipation linguistique est fréquente dans les collocations. Par exemple, le substantif " orage " est souvent en collocation avec le verbe "éclater " de même que le substantif « tempête " avec la locution verbale "faire rage ", etc. L'anticipation linguistique ne fait appel qu'à la connaissance d'une langue et à la fréquence d'utilisation des expressions figées.

Synonymes : ANTICIPATION DE LANGUE

ANTICIPATION SÉMANTIQUE

ANTICIPATION VERBALE 
ANTICIPATION SÉMANTIQUE

V. ANTICIPATION LINGUISTIQUE

ANTICIPATION SENSIQUE

V. ANTICIPATION DU SENS

ANTICIPATION VERBALE

V. ANTICIPATION LINGUISTIQUE

\section{APPRÉHENSION DU SENS}

Acte par lequel le sens d'un message est saisi au moyen d'un rapport qui s'établit entre " les engrammes de la connaissance linguistique (grâce auxquels les significations linguistiques sont reconnues sous les paroles entendues (ou sous les mots lus]) et les engrammes non verbaux de la connaissance (Seleskovitch $1975: 168$ ) ".

\section{ASSIMILATION DU SENS}

Résultat de la transformation de paroles entendues ou d'énoncés lus en un sens non verbal, c'est-à-dire dénué de formes linguistiques.

\section{BAGAGE COGNITIF}

Ensemble des connaissances et des expériences acquises par une personne, qui constituent son savoir permanent.

* Le bagage cognitif relève de la mémoire à long terme ; il est actualisé lors de la réception d'un discours ou d'un texte.

\section{COMPLÉMENTS COGNITIFS}

Éléments de connaissance mobilisés par un énoncé en même temps que les concepts attachés de façon stable aux signes linguistiques. Ils aident le traducteur ou l'interprète à comprendre un texte ou un discours et à en constituer le sens.

* Les compléments cognitifs qui interviennent dans la constitution du sens sont : l'auteur, le contexte verbal, le contexte cognitif, le contexte temporel et spatial, le destinataire, la situation et les connaissances thématiques pertinentes.

\section{CONCEPTUALISATION}

Action de constituer " un souvenir cognitif par intégration des fragments successifs de chaîne parlée [ou écrite] à des connaissances antérieures " (Lederer 1981 : 50).

\section{CONNAISSANCES PARTAGÉES}

Ensemble de connaissances thématiques dont disposent les interlocuteurs et qui leur permettent de communiquer de façon elliptique.

Synonyme : SAVOIR PARTAGÉ

\section{CONNOTATION}

Significations secondes apportées par la charge émotive d'un mot, variant avec chaque individu : elles s'ajoutent à la signification conceptuelle d'un mot et à sa charge émotive en langue. 


\section{CONTEXTE COGNITIF}

Informations que le déroulement du discours apporte à l'auditeur ou celui du texte au lecteur et qui interviennent dans sa compréhension.

* Le détail des informations qui constituent le contexte cognitif est retenu par la mémoire à court terme; leur synthèse enrichit le bagage cognitif.

\section{CONTEXTE VERBAL}

Ensemble des mots contenus dans la mémoire immédiate et qui correspondent à l'aspect formel de l'unité de sens.

* "Le contexte [verbal], c'est-à-dire la présence simultanée d'un ensemble de mots dans la mémoire immédiate, ... [correspond] dans l'écrit ... à l'empan de l'appréhension visuelle" (Seleskovitch, Lederer $1984: 44$ ).

V.a. MÉMOIRE IMMÉDIATE

UNITÉ DE SENS

\section{EMPAN DE LA MÉMOIRE IMMÉDIATE}

\section{EMPAN MNÉSIQUE}

\section{EMPAN MNÉSIQUE}

Contenance de la mémoire immédiate ; quantité d'informations qui peut être appréhendée et retenue momentanément et qui se situe autour de sept ou huit mots. Synonyme : EMPAN DE LA MÉMOIRE IMMÉDIATE

\section{V.a. MÉMOIRE IMMÉDIATE}

\section{FIDÉLITÉ D'UNE TRADUCTION}

Qualité d'une traduction définie par " sa valeur d'équivalence avec les sens exprimés par le texte original " et "par sa conformité à la stylistique de la langue dans laquelle elle s'exprime" (Seleskovitch dans Lederer $1981: 9$ ).

* "Le premier critère juge de son exactitude, le deuxième de son intelligibilité " (Ibid. $1981: 9$ ).

\section{MÉMOIRE À COURT TERME}

Mémoire différée caractérisée par la rétention de souvenirs d'une durée de quelques minutes à quelques heures.

V.a. MÉMOIRE DIFFÉRÉE

\section{MÉMOIRE À LONG TERME}

Mémoire différée caractérisée par la rétention de souvenirs d'une durée de plusieurs heures à plusieurs années.

V.a. MÉMOIRE DIFFÉRÉE

\section{MÉMOIRE AUDITIVE}

V. MÉMOIRE IMMÉDIATE

\section{MÉMOIRE COGNITIVE}

Mémoire à court terme caractérisée par une capacité de rétention de souvenirs déverbalisés ; les formes linguistiques disparaissent tandis que subsistent les souvenirs cognitifs.

Synonyme : MÉMOIRE CONCEPTUELLE

V.a. MÉMOIRE À COURT TERME 


\section{MÉMOIRE CONCEPTUELLE \\ V. MÉMOIRE COGNITIVE}

\section{MÉMOIRE DIFFÉRÉE}

Mémoire caractérisée par une rétention d'informations après que ces dernières ont été appréhendées.

La capacité de la mémoire différée est presque illimitée et la durée de rétention dépend de l'importance pour le sujet de ce qui a été mémorisé.

V.a. MÉMOIRE À COURT TERME

MÉMOIRE À LONG TERME

MÉMOIRE COGNITIVE

MÉMOIRE CONCEPTUELLE

\section{MÉMOIRE IMMÉDIATE}

Mémoire caractérisée par une capacité d'appréhension de sept ou huit vocables et par une durée de rétention, en l'absence de répétition, de deux ou trois secondes. * La mémoire immédiate de l'auditeur ou du lecteur ne retient les structures sonores des mots que le temps nécessaire à l'identification des significations appropriées.

Synonymes : MÉMOIRE AUDITIVE

\section{MÉMOIRE OPÉRATIONNELLE}

MÉMOIRE VERBALE

\section{MÉMOIRE OPÉRATIONNELLE}

V. MÉMOIRE IMMÉDIATE

\section{MÉMOIRE VERBALE}

\section{MÉMOIRE IMMÉDIATE}

\section{MOT TRADUISIBLE}

Tout mot correspondant à un concept délimité et dont le sens en discours est identique à sa signification dans la langue.

Synonyme : MOT TRANSCODABLE

V.a. TRANSCODAGE

MOT TRANSCODABLE

V. MOT TRADUISIBLE

\section{PRÉSENCE MNÉSIQUE}

«Rétention par la mémoire immédiate des quelques signes linguistiques qui, chez l'auditeur, constituent la face formelle de l'unité de sens et chez le locuteur l'expression verbale de son idée. " (Seleskovitch, Lederer 1984 : 41)

SAVOIR PARTAGÉ

\section{CONNAISSANCES PARTAGÉES}

\section{SENS (D'UN ÉNONCÉ)}

Signification pertinente de " tout énoncé destiné à un interlocuteur dans un contexte et une situation déterminés. 》 (Lederer 1981 : 114) 


\section{SENS (D'UN MOT)}

"Signification pertinente, telle qu'elle se dégage des significations linguistiques dans l'acte de parole, grâce au contexte et aux circonstances dans lesquels s'inscrit le signe. " (Seleskovitch $1975: 12$ )

\section{SENS PRAGMATIQUE (D'UN MOT)}

\section{SIGNIFICATION PRAGMATIQUE (D'UN MOT)}

\section{SENS PREMIER (D'UN MOT)}

Première signification à surgir à l'esprit lorsqu'un mot est lu ou entendu.

* Le sens premier d'un mot correspond à son acception la plus courante parmi plusieurs acceptions possibles.

\section{SIGNIFICATION (D'UN MOT)}

Aire sémantique recouverte par un mot hors contexte.

* La signification d'un mot correspond à la ou aux acception(s) qu'en donnent les ouvrages lexicographiques (dictionnaires, lexiques, glossaires, etc.).

\section{SIGNIFICATION CONTEXTUELLE (D'UN MOT)}

Traits sémantiques pertinents retenus dans le discours ou le texte par rapport à l'aire sémantique recouverte par un mot hors contexte.

V.a. SIGNIFICATION (D'UN MOT)

\section{SIGNIFICATION PRAGMATIQUE (D'UN MOT)}

Actualisation sémantique individuelle d'un mot dans un contexte donné.

* Bon nombre de mots sont porteurs d'une signification pragmatique qui leur est conférée par la parole individuelle.

Synonyme : SENS PRAGMATIQUE (D'UN MOT)

V.a. SIGNIFICATION CONTEXTUELLE (D'UN MOT)

\section{SIGNIFICATION (D'UN MOT)}

\section{SITUATION (INTERPRÉTATION)}

Ensemble des éléments non linguistiques perçus en même temps que le discours et qui permettent, lorsqu'ils sont pertinents, d'orienter la compréhension.

* Pour l'interprète, la situation correspond au cadre physique dans lequel il se trouve, c'est-à-dire à la salle où a lieu l'interprétation, à ce qu'il voit, aux gestes de l'orateur, etc.

\section{TRADUCTION}

1) Action de "faire passer le contenu d'un texte ou d'un discours [en langue $x$ ] dans un autre texte ou discours [en langue y]. " (Seleskovitch, Lederer 1984 : 136)

2) Résultat du passage du contenu d'un texte ou d'un discours en langue $x$ dans un autre texte ou discours en langue $y$.

\section{TRADUCTION LINGUISTIQUE \\ V. TRANSCODAGE 1)}




\section{TRANSCODAGE}

1) Opération qui a pour but d'établir des correspondances entre deux langues. Synonyme : TRADUCTION LINGUISTIQUE

2) Opération qui a pour but de transposer d'une langue à une autre tout mot ou toute expression pour lesquels il existe des correspondances établies.

Synonyme : TRANSPOSITION

V.a. MOT TRADUISIBLE

\section{TRANSPOSITION}

V. TRANSCODAGE 2)

\section{UNITÉ DE SENS}

Élément de sens qui subsiste après qu'un énoncé a été lu ou entendu et que s'est produite une réaction cognitive ; cet élément s'intégrera dans un ensemble plus vaste.

* L'unité de sens est déverbalisée ; elle apparaît à l'intérieur de l'empan mnésique, soit à l'intérieur d'un segment d'environ sept ou huit mots.

Synonyme : UNITÉ DE COMPRÊHENSION

V.a. EMPAN MNÉSIQUE

\section{VOULOIR-DIRE (D'UN LOCUTEUR)}

Sens qu'un locuteur veut transmettre à un interlocuteur au moyen d'un énoncé formulé dans un contexte et dans une situation donnés.

Note

* L'auteur remercie Danica Seleskovitch et Marianne Lederer d'avoir bien voulu relire son manuscrit et de lui avoir fait part de leurs commentaires.

\section{RÉFÉRENCES}

Ourrages généraux

Grand dictionnaire encyclopédique Larousse (1982-1985) : Paris, Larousse, 13 vol. parus.

Grand Larousse encyclopédique (1960-1964) : Paris, Larousse, 10 vol.

Petit Robert (1982) : Paris, Société du nouveau Littré, 2175 p.

Ouvrages spécialisés

BARBIZET, J. (1964) : Études sur la mémoire, 1re série, Paris, l'Expansion scientifique française, 52 p.

BARBIZET, J. (1967) : Études sur la mémoire, $2^{e}$ série, Paris, l'Expansion scientifique française, 59 p.

DELISLE, Jean (1980) : l'Analyse du discours comme méthode de traduction, Ottawa, Éditions de l'Université, 282 p.

DELISLE, Jean (1981) : "De la théorie à la pédagogie : réflexions méthodologiques ", dans Jean Delisle (réd.), l'Enseignement de l'interprétation et de la traduction : de la théorie à la pédagogie, Ottawa, Éditions de l'Université, pp. 135-151.

FLORÈS, César (1982) : la Mémoire, Paris, PUF, Coll. Que sais-je ?, 125 p.

LEDERER, Marianne (1973) : "La traduction : transcoder ou réexprimer? ", dans Études de linguistique appliquée, no 12 , pp. 7-25.

LEDERER, Marianne (1976) : «Synecdoque et traduction ", dans Études de linguistique appliquée, $\mathrm{n}^{\circ} 24, \mathrm{pp}$. 13-41.

LEDERER, Marianne (1981) : la Traduction simultanée, Paris, Minard Lettres modernes, 454 p.

LEDERER, Marianne (1982) : «Le processus de la traduction simultanée ", dans Multilingua, vol. 1, no 3 , pp. 149-158.

SELESKOVITCH, Danica (1968) : l'Interprète dans les conférences internationales, Paris, Minard Lettres modernes, $261 \mathrm{p}$.

SELESKOVITCH, Danica (1973) : "Vision du monde et traduction ", dans Études de linguistique appliquée, $n^{0} 12$, pp. $105-109$.

SELESKOVITCH, Danica (1975) : Langage, langues et mémoire, Paris, Minard Lettres modernes, 272 p. 
SELESKOVITCH, Danica (1976) : "Traduire : de l'expérience aux concepts », dans Études de linguistique appliquée, $\mathrm{n}^{\circ} 24$, pp. 64-91.

SELESKOVITCH, Danica et Marianne LEDERER (1984) : Interpréter pour traduire, Paris, Didier Érudition, $311 \mathrm{p}$. 